\title{
Soft X-ray response of X-ray CCD camera XIS onboard Astro-E2
}

\author{
Kiyoshi Hayashida ${ }^{a *}$, Ken’ichi Torii ${ }^{a}$, Masaaki Namiki ${ }^{a}$, Takayuki Shiroshoji ${ }^{a}$, Masayuki Shoji ${ }^{a}$, \\ Satoru Katsuda ${ }^{a}$, Daisuke Matsuura ${ }^{a}$, Tomofumi Miyauchi ${ }^{a}$, Hiroshi Tsunemi ${ }^{a}$, \\ Takayoshi Kohmura $^{b}$, Haruyoshi Katayama ${ }^{c}$ \\ ${ }^{a}$ Osaka University, 1-1 Machikaneyama-cho, Toyonaka, Osaka 560-0043, Japan; \\ ${ }^{b}$ Kougakuin University, 2665-1 Nakano-cho, Hachioji, Tokyo 192-0015, Japan; \\ ${ }^{c}$ Tsukuba Space Center/ JAXA, 2-1-1 Sengen, Tsukuba, Ibaragi 305-8505, Japan
}

\begin{abstract}
We present the current status of soft X-ray calibration of X-ray CCD cameras, X-ray Imaging Spectrometer (XIS), onboard Astro-E2. We perform soft X-ray calibration of four front illuminated (FI) CCD cameras and two back illuminated (BI) CCD cameras, among which four cameras will be selected to be installed on the satellite. The calibration aims to measure the quantum efficiency and re-distribution function of the CCDs as a function of incident Xray energy. A soft X-ray spectrometer is used to measure these items. In addition, we employ a gas proportional counter and an XIS engineering unit as reference detectors for the quantum efficiency measurement. We describe how we calibrate the absolute quantum efficiency of the XIS using these instruments. We show some of the preliminary results of the calibration including quick look results of BI CCD cameras.
\end{abstract}

Keywords: Astro-E2, XIS, X-ray CCD, calibration

\section{ASTRO-E2 XIS}

Astro-E2 is an X-ray astronomy satellite scheduled to be launched on February 2005. It is a recovery mission of AstroE1, which was lost during its launch in February 2000. Astro-E2 carries five sets of X-ray telescopes (XRT) and a hard $\mathrm{X}$-ray detector (HXD) as scientific instruments. One of the X-ray telescopes has an X-ray micro calorimeter (XRS: XRay Spectrometer) on its focal plane, and the other four telescopes have X-ray CCD cameras (XIS: X-ray Imaging Spectrometer). Wide energy range from $0.2 \mathrm{keV}$ to $600 \mathrm{keV}$ is covered by a combination of these instruments. At X-ray energy range below $12 \mathrm{keV}$, the XRS provides superior energy resolution of $6-7 \mathrm{eV}$. The XIS covers similar energy range to the XRS, from 0.2 to $12 \mathrm{keV}$, with moderate energy resolution but with higher sensitivity. Combined use of XRS and XIS will be effective to measure complex X-ray spectra in which narrow and broad lines are superposed on continuum emission. The XIS is also important as an X-ray imager, covering FOV of 19arcmin times 19arcmin. Furthermore, it should be noted that the XIS will be the only soft-X-ray detector on Astro-E2 available after the XRS life time is expired.

\subsection{Overview of Astro-E2 XIS}

The design of Astro-E2 XIS is basically the same as that for Astro-E1 described in Hayashida et al. ${ }^{1,2}$ The system consists of four X-ray CCD cameras, analog electronics (AE), and digital electronics (DE). Each CCD camera has one frame-transfer type CCD chip manufactured by MIT Lincoln Laboratory. The size of the CCD chip is $24 \mathrm{mmx} 24 \mathrm{~mm}$ for an imaging area, in which $1 \mathrm{M}$ pixels are included. Four readout nodes are equipped for the CCD chip, and each readout node outputs the pixel data from a quadrant of the CCD. The CCD is cooled down to $-90^{\circ} \mathrm{C}$ with a thermo-electric cooler. Nominal exposure time is 8 seconds, though various clocking modes are available by changing micro codes stored in the AE. Digitizing the pixel signal from the CCD readout, changing the DAC voltages of the clocks, controlling the CCD temperature, and collecting house keeping data are also the tasks of the AE. The digitized pixel data are transferred from the $\mathrm{AE}$ to the $\mathrm{DE}$, and the $\mathrm{DE}$ evaluates the dark level and picks up X-ray events from them. Various data formats are available for the DE output, which will be transferred to the ground through satellite's data processor.

Several modifications were introduced to the design of Astro-E2 XIS from that of Astro-E1 XIS, as described in Dotani et al. ${ }^{3}$ First, CCD chip model was revised from CCID-17 to CCID-41. CCID-41 is also manufactured by MIT

* Further author information: (Send correspondence to K.H.)

K.H.: E-mail: hayasida@ess.sci.osaka-u.ac.jp, Telephone: +81 (6) 68505476 
Lincoln Laboratory and has the same size and format as CCID-17. One of the modifications of CCID-41 from CCID-17 is implementation of a charge injection gate. The charge injection gate enables us to measure charge transfer efficiency using injected charge, which will be useful to evaluate radiation damage effect in orbit. Second, the width of gate overlap and channel stop was reduced, which will improve the low energy X-ray quantum efficiency. Third, the surface of the imaging area of CCID-41 is covered with a kind of glass, called BPSG, with thickness of about 250nm. In order to gain the low energy X-ray quantum efficiency, the BPSG layer was etched down to about 70nm. The camera body of the XIS has been slightly modified from the Astro-E1 design, too. The CCD supporting mechanism was modified so that one thermo-electric cooler (TEC) is employed per one CCD. We put two calibration sources inside the camera body and installed an additional calibration source on the backside of the door. The calibration source on the door will be used in some ground tests and in-flight calibration before the door open. The analog electronics are very similar to the ones for Astro-E1 but has a capability to drive the charge injection gate. The digital electronics are also very similar to the ones for Astro-E1, though some of the parts were no longer available and replaced with new ones.

Fabrications of the flight model cameras, AE, and DE were completed before August 2003. We connected the components at ISAS and performed various kinds of function tests. The XIS system joined the first integration test of the Astro-E2 satellite, and was transferred to Osaka University and Kyoto University, where calibrations were done, December 2003. We share the calibration task, low energy part $(0.2-2.2 \mathrm{keV})$ at Osaka and high energy part (above $1.5 \mathrm{keV}$ ) at Kyoto. Note that the calibration of the optical blocking filters (OBF) were already done as described in Kitamoto et al. ${ }^{4}$, and the OBF were not installed in the XIS cameras during the calibration phase at Osaka and Kyoto. The XIS system will go back to ISAS on July 2004, and will be onboard the satellite and join the thermal vacuum test of the satellite on August 2004.

\subsection{Backilluminated CCD for Astro-E2 XIS}

We started the Astro-E2 XIS program by employing front-illuminated (FI) device of CCID-41 for all the four XIS cameras. In fact, the first integration test was completed with the four FI CCD cameras. However, in the course of the program, we decided to introduce back-illuminated (BI) CCD chips and fabricated two XIS flight model cameras with BI-CCDs. Note that FI-CCD cameras and BI-CCD cameras have the same electric and mechanical interfaces, and the same AE and the DE can be used. We have not yet fixed how many (one or two) FI-CCD cameras will be replaced with the BI-CCD cameras, but we completed the soft X-ray calibration of the four FI-CCD cameras at Osaka before April 2004. Calibration of the two BI-CCD cameras is done on June 2004. In this paper, we report the soft X-ray calibration at Osaka, mainly on that for the FI-CCD cameras, but mention some quick look results on BI-CCD cameras. Note that the results presented here are not the final ones but preliminary ones, for which we need further analysis.

\section{SOFT X-RAY CALIBRATION OUTLINE}

We used a soft X-ray calibration system consisting of an X-ray generator and a grating spectrometer as shown in Fig.1(a). The system is installed in a clean-room at Osaka University and was used for Astro-E1 XIS calibration. This system enables us to obtain CCD responses against continuous X-ray energies simultaneously. The goal of our calibration is to make response matrices of the XIS. The response matrices comprises a quantum efficiency (QE) and a re-distribution function against single energy incident X-rays. The latter may be represented by the average pulse-height $(\mathrm{PH})$ and the energy resolution as functions of the incident X-ray energy (Ex), though deviation of the re-distribution function from Gaussian is needed to be taken into account. In order to measure the re-distribution function, incident X-ray energies must be known accurately. Various characteristic X-ray lines on the continuum X-ray spectrum are available for it. On the other hand, the intensity of the incident X-rays, which is essential for the QE measurement, is not easy to be obtained. We take several steps employing two kinds of reference detectors, a gas proportional counter and an XIS engineering unit. In this section, we outline the calibration system and experiments with it.

\subsection{Soft $X$-ray source and grating spectrometer}

The soft X-ray calibration system and its usage for Astro-E1 XIS calibrations were described in series of papers ${ }^{1,25,6}$. The system consists of a soft X-ray source, Manson model 2 ultra soft X-ray source, and a grating spectrometer by Hetrick Science, and a chamber in which a CCD camera and/or a gas proportional counter are installed. Continuum X-rays from the X-ray source are dispersed with the spectrometer and irradiated to a CCD installed in the chamber. Incident X-ray energies are known from the positions of X-ray events on the CCD along their dispersion direction, enabling us to obtain 
$\mathrm{X}$-ray responses against continuous X-ray energies simultaneously. Although higher order X-rays contaminate to the first order X-rays for each dispersion position, they can be principally distinguished from their pulse heights.

We employed two kinds of grating elements, called SA and SX. The SA grating has 480lines/mm and optimized for $0.5 \mathrm{keV}$ X-rays, while the SX grating has 1440 lines $/ \mathrm{mm}$ and is optimized for $1.8 \mathrm{keV} \mathrm{X}$-rays. We primarily used the SA grating for $0.2-2.2 \mathrm{keV}$ range calibration but employed the SX grating to see detailed structures of the response around $\mathrm{Si}-\mathrm{K}$ edge. X-ray dispersion direction is vertical, and we need to change CCD position vertically by moving a stage on which XIS camera is installed, in order to cover the whole energy range. We nominally operated the soft X-ray source with an applied high voltage of $5 \mathrm{kV}$ and an emission current of $0.7 \mathrm{~mA}$, as we did for Astro-E1 XIS calibration. In some cases for the Astro-E2 XIS calibration, we used $1 \mathrm{kV} / 0.3 \mathrm{~mA}$ set in order to reduce X-ray intensity and to minimize the contaminations from higher order $\mathrm{X}$-rays. The $\mathrm{X}$-ray intensity was also tuned by changing the width of a slit installed at the exit of the X-ray source.

The emission current is always monitored with a current meter and found to be stabilized within $1 \%$ or less. Nevertheless, it is revealed that the X-ray intensity and spectrum varies in time even for the same emission current. The variation is a long term effect and most likely due to accumulation of tungsten, which makes a filament, on the surface of a silver anode. We repeated measurements with the same CCD detector, XIS engineering unit, in order to track this effect. We will report some detail on this variability of the soft X-ray source elsewhere.

In order to do simultaneous monitor of the X-ray intensity, we introduced a Si-PIN photo diode, IRD AXUV-100, with its amplifier AXUV-100HYBV. We placed the photo diode in the same chamber but on a different movable arm. When we place the photo diode at the position where 0th order X-rays come to, the diode amplifier outputs $\mathrm{mV}$ level signal to a full range of $10 \mathrm{~V}$. The dispersed $\mathrm{X}$-rays are too weak to be detected with this photo diode, indicating X-ray spectral change cannot be tracked with it. Although further improvements might be possible, the sensitivity of the photo diode system is too low for our calibration purpose currently.
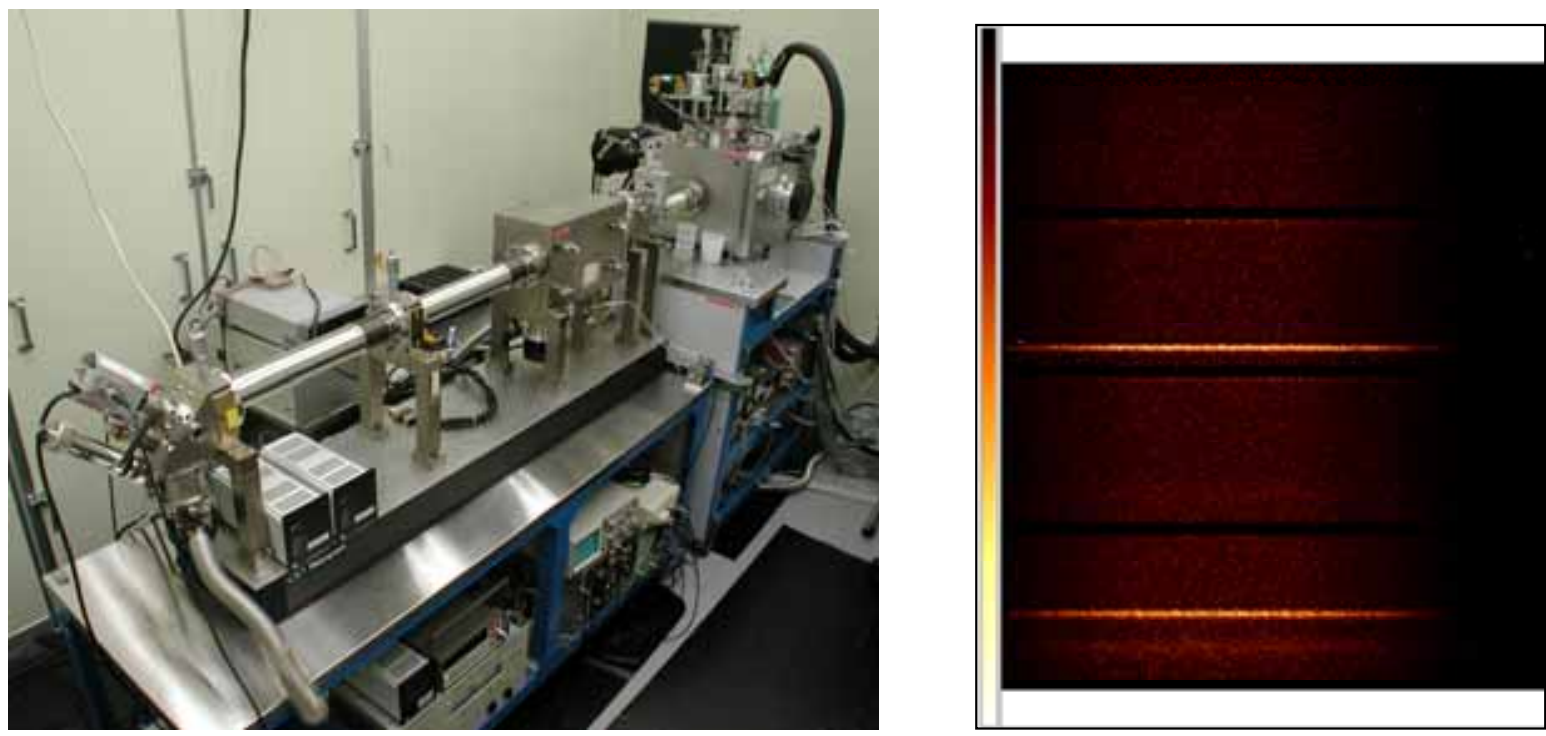

Figure 1. (a) Soft X-ray calibration facility in a clean room at Osaka University. From left, Manson ultra soft X-ray source, Hetrick spectrometer, and calibration chamber are seen. (b) X-ray CCD image (number of events detected in the CCD) with irradiation of dispersed X-rays with this system. The dispersion direction is vertical and the CCD serial register is set along that direction. Two bright horizontal lines correspond to O-K emission line of the first order light (middle of the image) and the second order light (bottom of the image), respectively The first order C-K line is also visible at the bottom of the image. Three dark lanes are artificially displayed to emphasize the boundary of four quadrants in an XIS CCD. There is no gap physically in the CCD.

\subsection{Outline of soft $X$-ray calibration of XIS}

We did soft X-ray calibration of XIS four flight model (FM) cameras with FI-CCD from December 2003 to April 2004. Calibrations of two XIS FM cameras with BI-CCD are performed in June 2004. Although we will eventually pick up four XIS FM cameras from these six, the calibration were done almost equally for them. In addition to these XIS FM 
cameras, we have an XIS engineering unit (EU) camera in which an FI-CCD is installed and the structure and function is the same as those of the XIS FM cameras. We make this XIS EU camera as a reference counter throughout a series of XIS FM calibrations.

As noted above, we have to know the intensity and the spectrum of the incident X-ray beam from the spectrometer. We need a reference X-ray detector of which quantum efficiency was absolutely calibrated. A gas proportional counter was employed for it in our calibration. We describe some detail of absolute calibration of the gas proportional counter (PC) in the next section. Next, as the second step, relative quantum efficiency of the XIS EU to this gas PC was calibrated by irradiating the same X-ray beam to these two alternately. In this case, we installed the XIS EU and the gas PC in the same chamber but on different movable stages to irradiate X-rays by turns. By this alternate measurement, we obtain the XIS EU quantum efficiency, in other word, the XIS EU become the second reference detector. Finally, as the third step, the X-ray beam was irradiated to the XIS EU and to one of XIS FM in order to get relative quantum efficiency of the XIS FM to the XIS EU. However, since only one camera can be installed in our calibration chamber at one time, we have been repeating the measurements with the XIS EU on various occasions between XIS FM measurements. Those repeated measurements revealed the long term variation in incident X-ray spectrum and intensity as mentioned above. Note that correction to the long term variation is possible by these repeated measurements with the XIS EU, though the results described in this paper are not necessarily corrected against that effect.

Because our gas PC is not position sensitive, we employed a slit to limit the width (thus energy) of the incoming beam onto these counters when we use the gas PC. Therefore, the absolute quantum efficiency of the gas PC and the relative quantum efficiency of the XIS EU to the gas PC are measured at several discrete energies. On the other hands, we don't use such a slit when X-rays are irradiated to whole the CCD area of the XIS EU or of the XIS FM. The relative quantum efficiency of each XIS FM to the XIS EU is thus measured at almost continuous X-ray energies from 0.2 to $2.2 \mathrm{keV}$. We don't perform alternate measurement of the gas PC and the XIS FM, primarily because we would avoid an emergency that the gas PC window break and sudden pressure increase in the chamber might damage the XIS FM. X-ray irradiation to whole the CCD area without using a slit is also effective to see detailed spectral structures as shown below.

As mentioned above, we basically used the SA grating with $\mathrm{X}$-ray source high voltage of $5 \mathrm{kV}$ for FI-CCD calibrations. However, for BI-CCD calibration, we used reduced high voltage of $1 \mathrm{kV}$ with the SA grating for $0.2-0.8 \mathrm{keV}$ range in order to accommodate with higher quantum efficiency of the BI-CCD. For higher energy range calibration of $\mathrm{BI}-\mathrm{CCD}, 0.8-2.2 \mathrm{keV}$ range, we used the SX grating and applied high voltage of $5 \mathrm{kV}$.

\subsection{Operation of XIS in calibration experiments}

There installed three movable stages in the calibration chamber. The first stage, nearest to the beam entrance, is for a slit and a photo-diode. We set a gas PC or an ${ }^{55} \mathrm{Fe}$ calibration source on the second stage. The third stage is a cold plate on which an XIS camera is installed. The cold plate is connected to a liquid nitrogen dewar and is temperature controlled with heaters, typically $-60^{\circ} \mathrm{C}$ to $-40^{\circ} \mathrm{C}$. The XIS CCD is further cooled down to $-90^{\circ} \mathrm{C}$ with a thermo-electric-cooler (TEC) installed inside the XIS camera. One thermal cycle, from cooling start with liquid nitrogen to cooling stop, is no longer than one day, to avoid possible accumulation of contaminants on CCD surface. We estimate the amount is less than $30 \mathrm{~nm}$ from a TQCM measurement prior to the calibration.

Analog electronics and temperature control electronics (AE/TCE) are set outside the calibration chamber. AE/TCE and XIS cameras have one to one correspondence, primarily for external registers used to tune the injection charge amount, and we basically followed correct combinations. Although the AE/TCE has a large flexibility to clock the XIS CCD, we used a normal clocking mode, and a parallel-sum (P-sum) clocking mode in the calibration. The normal clocking mode is a usual timed exposure with an exposure time of 8 seconds. The number of parallel summation in the P-sum mode is flexible but was set to be 128 during the calibration experiments. Charge injection function was also tested, though not described in this paper. The AE/TCE is connected to electric ground support equipment (EGSE), which consists of an electric interface box and DSP cards installed in a Sun workstation. The EGSE and software in Sun WS simulate functions of the XIS DE.

In the calibration experiment, all the CCD data are stored in hard disks as frame format FITS files. Delay look analysis simulating the data reduction process in the XIS DE is applied to the stored FITS files. The delay look analysis includes some data reduction process which will be done on the ground even after the system is in orbit. Optimization of parameter values used in these data reduction process is one of the aims of calibration experiments. Nevertheless, the optimization is not completed, and the results presented here are not necessarily the products with optimized parameters. 


\section{STEP1: CALIBRATION OF GAS PROPORTIONAL COUNTER}

We employed a gas flow type proportional counter (PC) as a reference for the quantum efficiency measurement. The gas PC has a single anode in a single gas cell of which thickness is $40.9 \mathrm{~mm}$. We operate the PC with $1 \mathrm{~atm}$ P10 (Ar90\%: $\mathrm{CH}_{4} 10 \%$ ) gas. The entrance window is made of a mesh supported polypropylene film, of which X-ray transmission was measured before constructing the PC in 1999 by Shouho $^{7}$. The transmission measurement was done by on-off method comparing the X-ray intensity with and without inserting the PC window in front of a CCD. The same spectrometer system described above was used for this measurement. The measured transmission at X-ray energy range from $0.4 \mathrm{keV}$ to $2.2 \mathrm{keV}$ was fitted with a model consisting of a polypropylene film of $1.49 \pm 0.03 \mu \mathrm{m}$ and water of which equivalent thickness is $0.17 \pm 0.02 \mu \mathrm{m}$, and supporting mesh transmission of 0.756 . It was not necessarily be water but $\mathrm{O}$ $\mathrm{K}$ edge was visible in the transmission curve. With this window transmission and geometrical thickness of the gas cell, absolute quantum efficiency of the gas PC can be calculated as was done by Shouho ${ }^{7}$. Nevertheless, because the transmission measurement did not cover the energy range below $0.4 \mathrm{keV}$ or statistical error was large below $0.5 \mathrm{keV}$, and because four years passed since the transmission measurement, we performed an independent measurement of the quantum efficiency of the gas PC. We applied a newly developed slant incident method for it.

\subsection{Slant Incident Method to Measure Absolute Quantum Efficiency of Gas Proportional Counter}

If we simplify an X-ray detector with a surface dead layer and a detection layer, the detection efficiency against slant incident X-rays is different from that for a straight incidence, which is modeled with a cosine factor of the incident angle. If the ratio of detection efficiencies against two or more incident angles is obtained as a function of X-ray energy, the thicknesses of the dead layer and detection layer are evaluated from that. We proposed a calibration method based on this idea, called slant incident method, for X-ray CCDs ${ }^{8}$. We apply this method to our gas PC here.
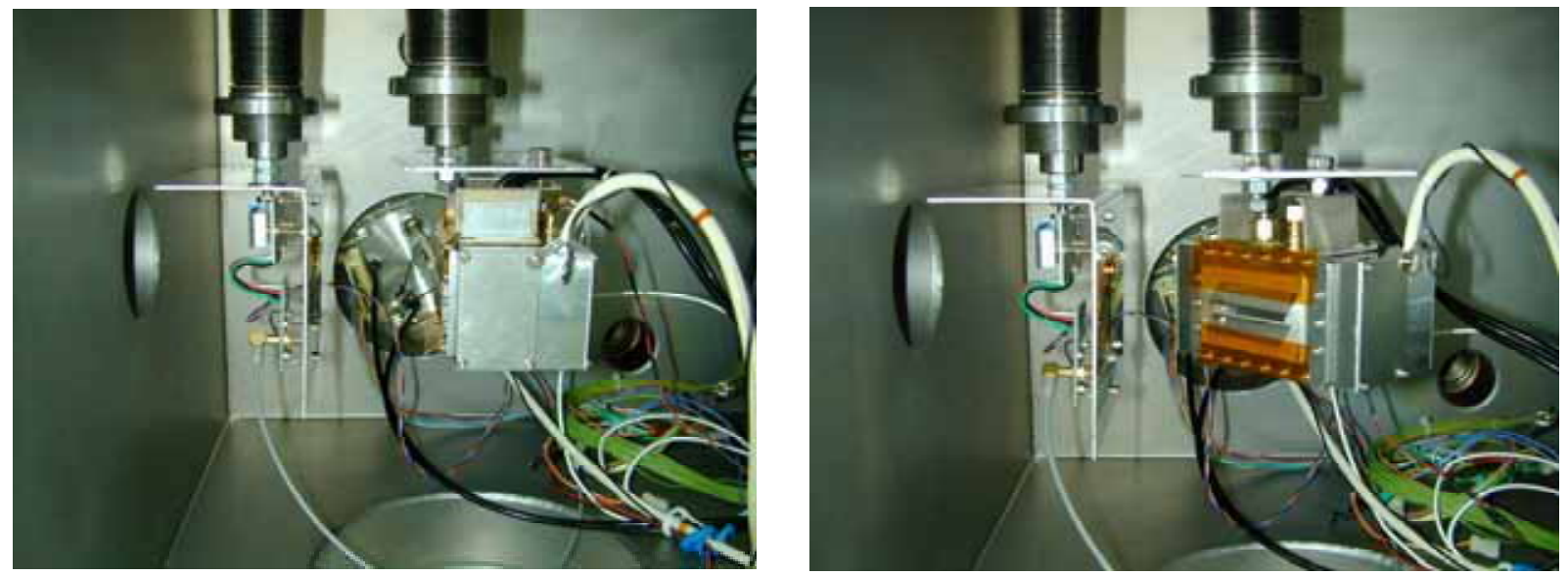

Figure 2. Gas proportional counter installed on the second movable stage in the chamber. X-rays through the spectrometer come from the duct on the left side wall. A slit is installed on the first movable stage. In the left panel, the gas PC is set for straight incidence, while it is set for $45^{\circ}$ slant incidence in the right panel. All the X-rays through the slit enter the gas PC entrance window in either case.

We employed the same soft X-ray source plus grating spectrometer system described above. A slit, $2 \mathrm{~mm}$ in height and $10 \mathrm{~mm}$ in width, was placed on the first movable stage in the calibration chamber and our gas PC on the second movable stage. We first set the gas PC so that the X-ray beam through the slit is irradiated at straight incidence (Fig.2 Left). X-ray spectra of several X-ray energies, the slit position and the PC position were adjusted accordingly, were obtained. Next we tilted the gas PC so as to realize $45^{\circ}$ slant incidence and repeated taking X-ray spectra (Fig.2 Rightt). Finally, we also did experiments with $30^{\circ}$ slant incidence. The X-ray spectra obtained include higher order X-rays, and the detected X-ray intensities of each component are derived by fitting the spectra, though separation is not necessarily clear for all the higher order components with gas PC resolution. Eleven energy points from $0.277 \mathrm{keV}$ to $1.775 \mathrm{keV}$ are available as a result. The ratio of the detected counting rate of $45^{\circ}$ incidence to $0^{\circ}$ incidence is plotted in Fig.3(a). For the 
X-ray energy considered here the X-ray detection efficiency is principally determined by the transmission of entrance window, and the ratio $45^{\circ} / 0^{\circ}$ should be less than one, as seen in the Fig.3(a).

Before fitting this plot with a model, we have to consider the effect of the supporting mesh of our gas PC window. The supporting mesh is made of stainless steel and the transmission should be almost constant in the soft X-ray energy range we are concerning. Therefore, it is hard to evaluate the mesh transmission from the slant incident method. We thus independently measured the transmission of a mesh that came from the same production lot as the mesh used in our gas PC. The transmission was $0.760 \pm 0.004,0.767 \pm 0.003,0.762 \pm 0.002$ at $0.525 \mathrm{keV}, 1.050 \mathrm{keV}, 1.775 \mathrm{keV}$, respectively. Averaging these, we adopt the mesh transmission of 0.763 . This value similar to the value determined from the window transmission measurement 0.756 , and consistent with the microscope measurement, $0.05 \mathrm{~mm}$ width lattice of $0.5 \mathrm{~mm}$ pitch. The thickness of the mesh is also $0.05 \mathrm{~mm}$ and we have to consider shadowing effect against slant incidence, though it is a minor effect.
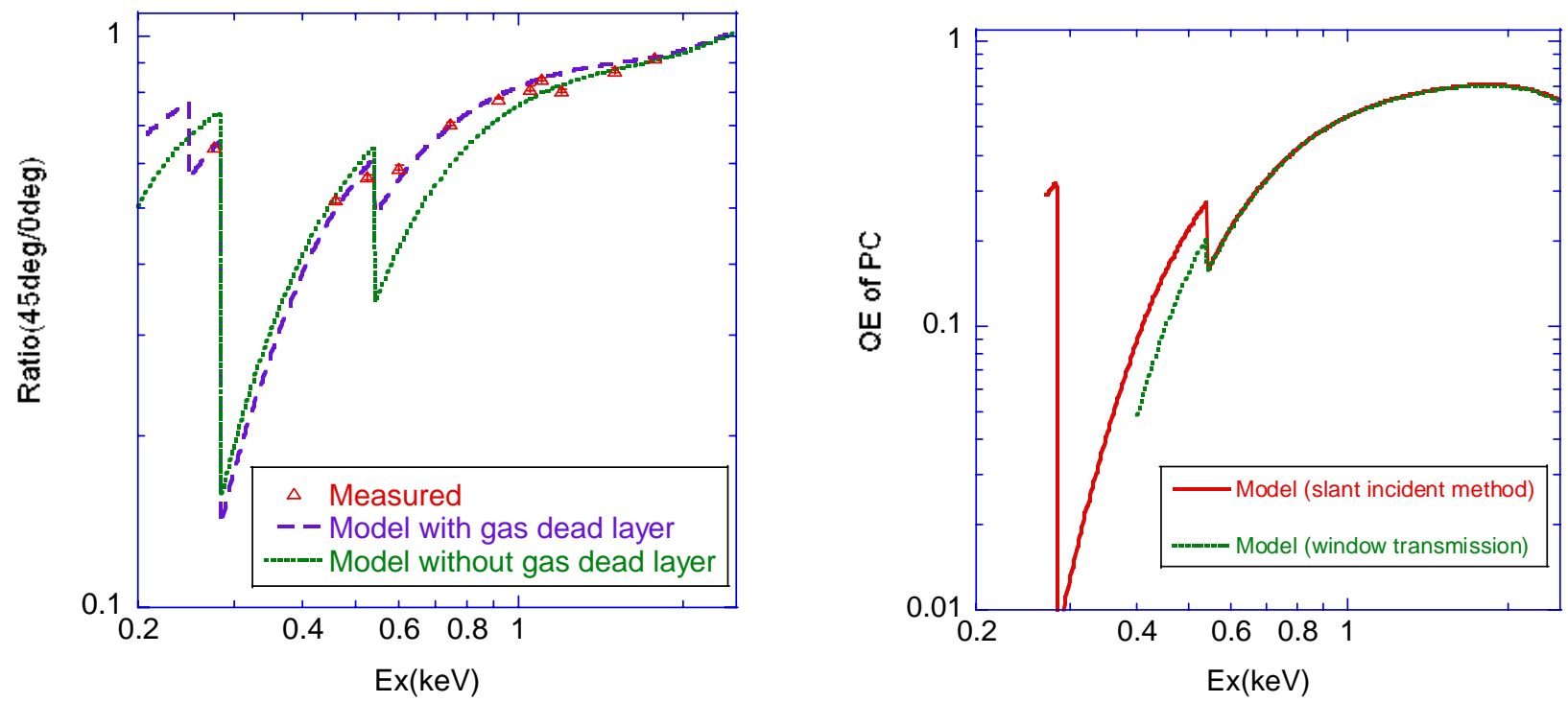

Figure 3. (a) Ratio of detected counting rate for $45^{\circ}$ to that for straight $\left(0^{\circ}\right)$ incidence. The dotted line indicates a model in which gas dead layer is not taken into account. The model hardly reproduces the data points. On the other hand, if we introduce a dead layer in the gas cell just beneath the window, such a model can reproduce the data as shown with a dashed line. (b) Quantum efficiency model of the gas PC. The Solid line represents the model based our slant incident method described in this paper, while the dotted line is a model from window transmission measurement by Shoho ${ }^{7}$ in 1999 the latter model the gas dead layer is not considered.

\subsection{Modeling the Absolute Quantum Efficiency Considering Gas Dead Layer}

The ratio of the detected X-ray counting rate $45^{\circ} / 0^{\circ}$ is fitted with a simple model considering polypropylene window with water contamination and the fixed gas cell of Argon gas. The best fit curve is displayed as a dotted line. It is apparent that the model cannot reproduce the measured data points. Note that if we neglect the data point of $0.277 \mathrm{keV}$, corresponding to C-K emission line and not included in the window transmission measurement by on-off method, the fit might be improved. Instead, we propose to consider an additional dead layer in the P10 gas just beneath the polypropylene window. Introducing this gas dead layer can reproduce the data points as shown Fig.3(a) with a dashed line. The best fit parameters for thicknesses are $0.974 \pm 0.017 \mu \mathrm{m}$ for polypropylene in the window, $0.305 \pm 0.014 \mu \mathrm{m}$ for water in the window, $71.6 \pm 1.2 \mu \mathrm{m}$ for the gas dead layer. As mentioned above, it is not necessarily water that attach to the window, but something including oxygen is needed to explain the data points. We can fit the ratio $30^{\circ} / 0^{\circ}$ with a same form model, which provided the thickness parameters to be $0.979 \pm 0.043 \mu \mathrm{m}, 0.367 \pm 0.035 \mu \mathrm{m}, 123.1 \pm 3.2 \mu \mathrm{m}$. We need to examine this difference later but adopt the results from $45^{\circ} / 0^{\circ}$ in this paper, because of its higher sensitivity.

Using these values for the window and dead layer thickness, we can model the absolute quantum efficiency (against straight incidence) of the gas PC as a function of X-ray energy, as shown in Fig.3(b) with a solid line. In the same figure, 
we plot the quantum efficiency modeled from the previous measurement of the window transmission ${ }^{7}$. The agreement above $0.6 \mathrm{keV}$ is excellent, while there found a discrepancy below $0.5 \mathrm{keV}$. The discrepancy is mainly due to the fact that we include the gas dead layer in the model while the transmission measurement did not. Apparently the gas dead later, if it exists, is impossible to be detected in the window transmission measurement. We thus adopt the model based on our slant incident method for the gas PC quantum efficiency. Nevertheless, since overall accuracy of the quantum efficiency calibration is determined by this gas PC calibration, we plan to perform further experiments with this gas PC after all the XIS calibration experiment.

\section{STEP2:CALIBRATION OF XIS EU AGAINST GAS PC}

The next step of the calibration it to measure the relative quantum efficiency of the XIS EU to the gas PC. In this measurement, we placed both detectors in the calibration chamber and irradiate X-rays to them through the slit alternately (Fig,4). For each slit position, we took hundreds frames of CCD data and a few thousands seconds exposure gas PC data. The soft X-ray source high voltage was set to be $5 \mathrm{kV}$, and the SA grating was used, although we are planning to perform similar experiment with other operation parameters after we finish the FM calibration experiment. We applied CCD data processing software, XISDL, to the frame data we obtained, in order to estimate and subtract the dark level and to extract X-ray events. We used a standard event extraction method using event grade, which was used for ASCA data analysis and will be used for Astro-E2 data analysis. We set the event threshold to be 50ADU and the split threshold to be 20ADU for FI-CCDs including the XIS EU, where 1ADU corresponds to about 3.6-4eV, depending on cameras and segments. We principally employ grade0,2,3,4,6 events in the analysis.
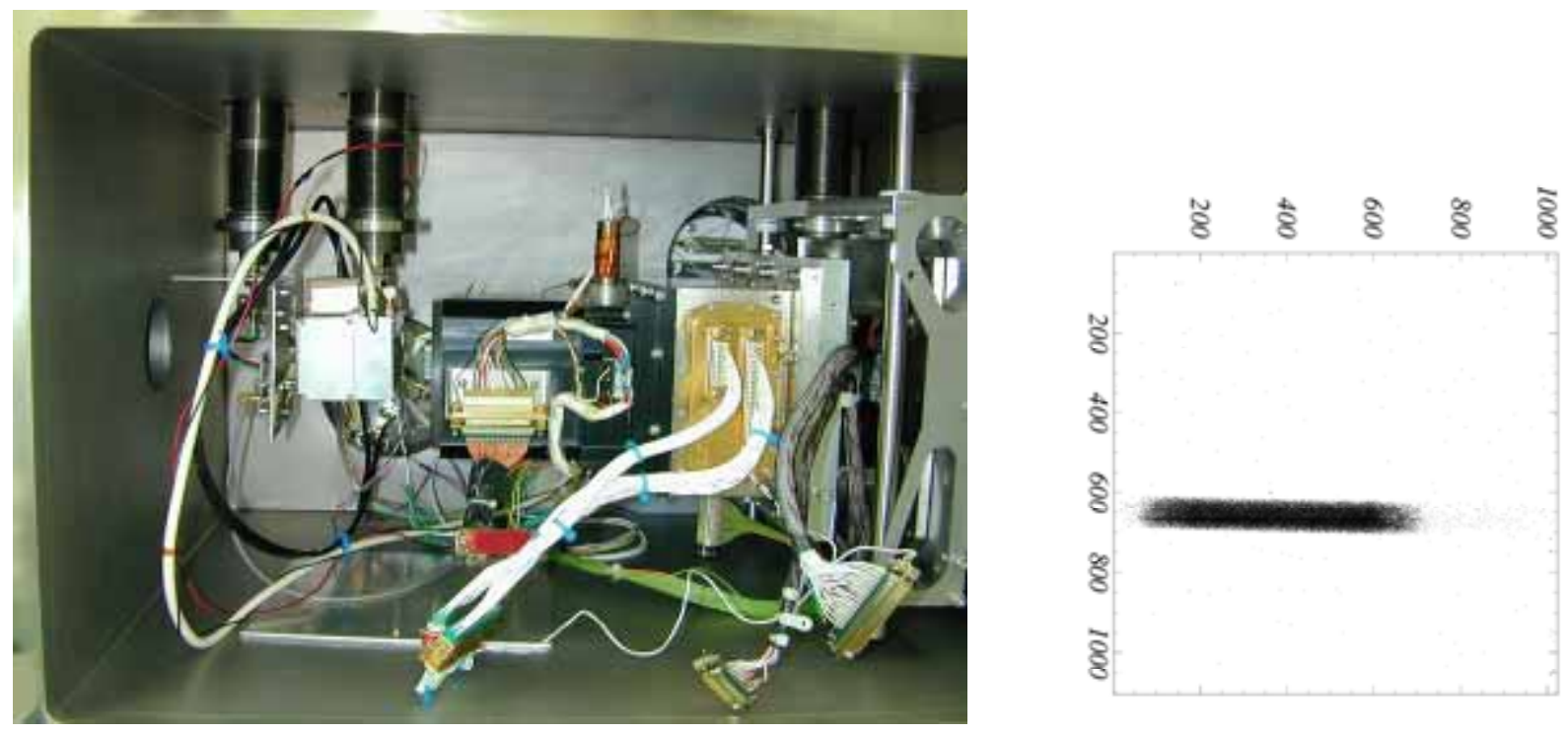

Figure 4. (a) XIS EU and gas PC installed in the calibration chamber. The XIS EU is set horizontally on the cold plate which is connected to the LN2 dewar inside the chamber. The gas PC, and a slit is on the first and the second movable stage, respectively. Xrays from the spectrometer through the slit are irradiated to the gas PC or the XIS EU alternately. (b) X-ray image taken with the XIS EU. We adjusted the slit location so that target X-ray energy is obtained. There are a small number of events which come during the parallel transfer.

Example of the X-ray spectra obtained are shown in Fig.5. Higher order X-rays are clearly separated in the XIS EU spectra (Fig.5(b)), and the counting rates for each component are easily derived. On the other hand, we need to fit the spectra of the gas PC (Fig.5(a)) to derive the corresponding counting rate. Multiplying the modeled quantum efficiency of the gas PC to the ratio of the derived counting rate of the XIS EU to that of the gas PC, the quantum efficiency of the XIS EU is derived as shown in Fig.6.

The quantum efficiency of the XIS EU is fitted with a model considering the XIS CCD structure, a residual BPSG layer on the surface, a Si electrode, a SiO2 insulator layer, and a Si depletion layer. We consider the overlap of the electrode and insulator to the adjacent ones, and also the channel stop structure. The overlap and the channel stop width were designed to be narrower for CCID-41 than CCID-17. We here assume the area fraction of the overlap and that of 
the channel stop to be $21 \%$ and $19 \%$, respectively. We also assume the depletion layer thickness to be $65 \mu \mathrm{m}$, which will be determined later with higher energy data. The best fit parameters of the fitting are, BPSG layer plus insulator layer thickness of $0.578 \pm 0.048 \mu \mathrm{m}$, Si electrode thickness to be $0.295 \pm 0.027 \mu \mathrm{m}$. As seen in the Fig, the fit is not perfect, and further tuning of the model is needed. Note also that near edge structures of O-K and Si-K in the quantum efficiency curve have not yet been taken into account, which should be included.
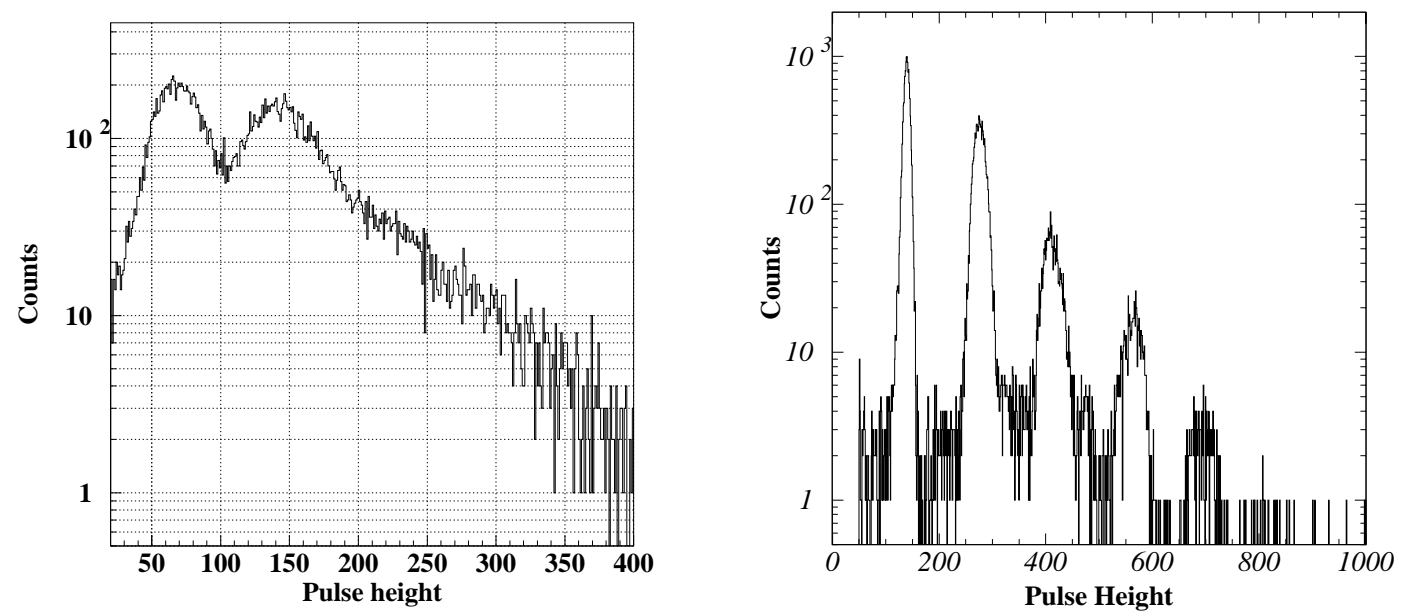

Figure 5. (a) X-ray spectrum measured with the gas PC in a setup shown in Fig.4 (a). The slit is placed so that the first order lights of $\mathrm{O}-\mathrm{K}$ emission line $(0.525 \mathrm{keV})$ pass through. The first and the second order peak is visible in the spectrum. (b) X-ray spectrum measured with the XIS EU CCD. The slit is fixed to the same position to that for Fig.5(a). Upto 5th.order lights are visible in the spectrum, though we only use the first order and the second order light in this case.

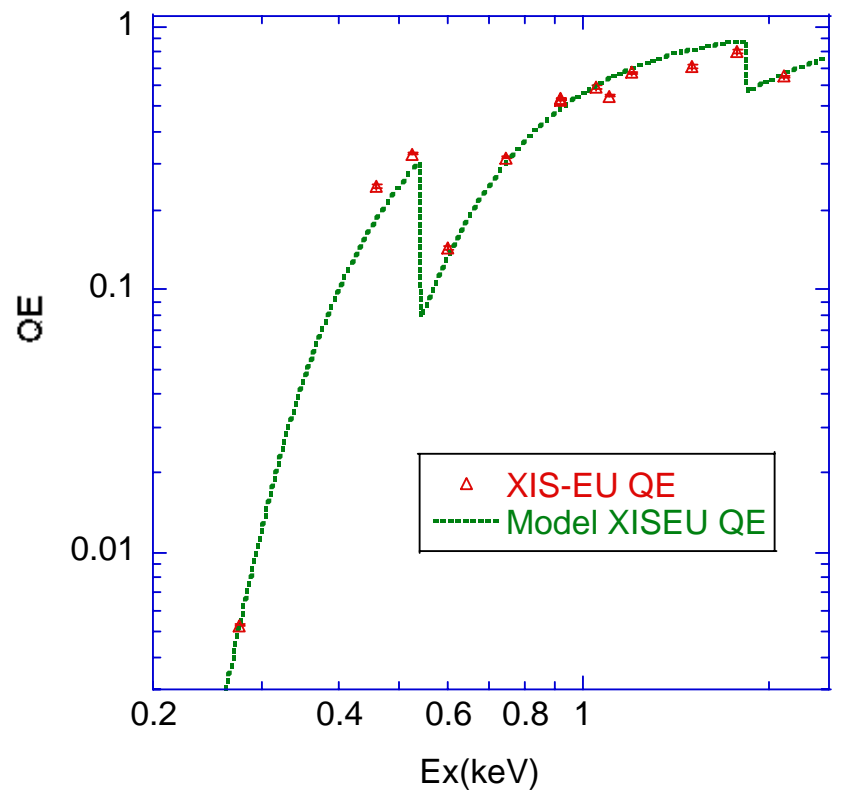

Figure 6. Quantum efficiency of the XIS EU CCD in soft X-ray range. Triangles are measured data points, i.e., measured relative quantum efficiency of the XIS EU to the gas PC multiplied by the modeled quantum efficiency of the gas PC, which is shown in Fig.3(b). Note that near edge structures around O-K and Si-K are not taken into account here. The dotted line indicates the best fit model for the XIS CCD quantum efficiency. See text in detail. 


\section{STEP3: CALIBRATION OF XIS FM CAMERAS AGAINST XIS EU}

After two steps of experiments, the XIS EU is ready to be used as a reference detector for the XIS FM calibration. The calibration items are not only the relative quantum efficiency to the XIS EU but also the Ex-PH relation and the ExFWHM relation. All these items are measured at continuous X-ray energies in the experiments in which dispersed Xrays from the spectrometer are irradiated at whole area of the XIS CCD. The slit to limit the X-ray energy used in the gas PC experiment was not employed in these experiments. We repeated such slitless measurements with the XIS EU before and after the XIS FM measurements in order to monitor the long term change in the incident X-ray beam. We have not yet completed the analysis nor experiments, but present some of the preliminary results here.

\subsection{Quantum Efficiency}

Relative quantum efficiencies of the XIS FM against the XIS EU are obtained simply by comparing the number of detected X-ray events at each CCD column, since the dispersion direction is almost parallel to the serial register. When we count the X-ray events at each CCD column, we limit the pulse height range so that only the first order X-rays are included. Using the XIS EU quantum efficiency model derived in the previous section, the quantum efficiencies of the XIS FM cameras are obtained. Fig.7(a) is an example of those. The quantum efficiencies of the XIS FM FI CCD cameras are slightly higher than that of the XIS EU at low energy side, which was qualitatively expected from thicker residual BPSG layer for the XIS EU. Note that near edge structures have not yet been included in the quantum efficiency in this figure, as for that of the XIS EU. The structure is visible when we plot the detected counting rate as a function of $\mathrm{X}$-ray incident energy around oxygen $\mathrm{K}$ edge as shown in Fig.7(b). We plan to make an empirical model for this structure and a less evident structure around the silicon K edge.
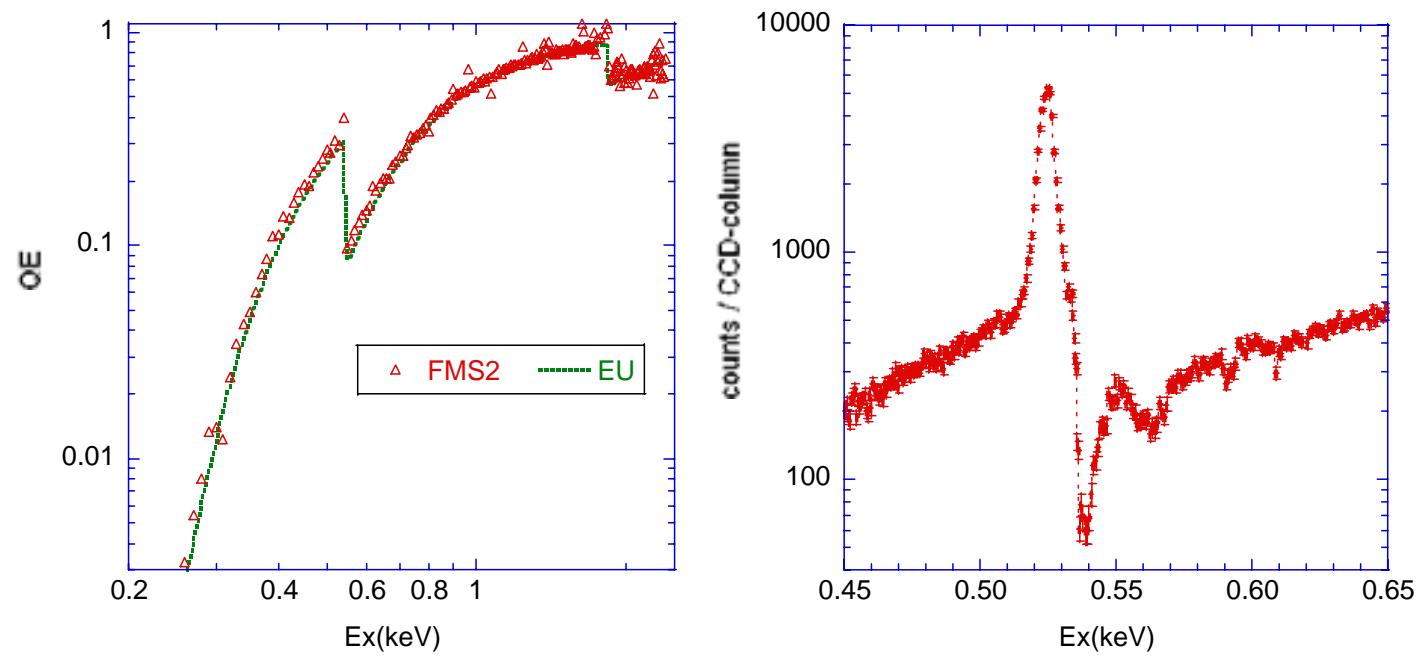

Figure 7. (a) Soft X-ray quantum efficiency of an FI-CCD in a XIS FM camera. The quantum efficiency model of the XIS EU is shown with a dotted line, as for Fig.6. Slightly higher quantum efficiency of this XIS FM than the XIS EU is visible. Note that we don't use optical blocking filters in XIS cameras during the calibration at Osaka, thus the quantum efficiency in this paper does not include the absorption by the OBF. (b) Detected X-ray counting rate around O-K edge with an XIS FI-CCD camera. The spectral features above the absorption edge is principally due to absorption structures by oxygen in solid, known as XANES. The structure should be taken into account the quantum efficiency model but has not yet been.

\subsection{Ex-PH Relation and Ex-FWHM Relation}

The pulse height $(\mathrm{PH})$ distribution extracted for each CCD column represents a response against single energy incident $\mathrm{X}$-rays, if we focus on the $\mathrm{PH}$ range where the first order $\mathrm{X}$-rays dominates. By fitting the $\mathrm{PH}$ distribution with a Gaussian profile, the center of pulse height and its width (FWHM) are obtained. It leads to an EX-PH relation and an EX-FWHM relation as shown in Fig.8(a). The Ex-PH relation is fitted with a straight line with nearly a zero offset. There found a deviation from the straight line at around and above the Si K-edge. Similar deviation was found in the XIS for Astro-E1, but the amount of deviation was reduced if we refine the data reduction, for example, taking into account 
of the detailed pulse profile including the tail structure ${ }^{1,2}$. Further examination of such effect for the Astro-E2 XIS results is needed The slope of the Ex-PH relation represents the gain of the XIS system. It ranges from $3.6 \mathrm{eV} / \mathrm{ADU}$ to 4.0 eV/ADU. From the Ex-FWHM relation, it is found that the energy resolution to $0.5 \mathrm{keV} \mathrm{X}$-ray incidence is as good as $40 \mathrm{eV}$. The energy resolution to $5.9 \mathrm{keV} \mathrm{X}$-ray incidence is typically $130 \mathrm{eV}$ or less.

In addition to these two relations, detailed response profile was examined. Fig. 9 shows the response against O-K line $(0.525 \mathrm{keV})$ incidence. The PH distribution has some distortion and a low energy tail, which was observed for Astro-E1 but the amount of the tail component is much smaller than that for Astro-E1. It might be related to design change of the electrode, e.g., narrower channel stop, but needs further examinations at different X-ray energies.
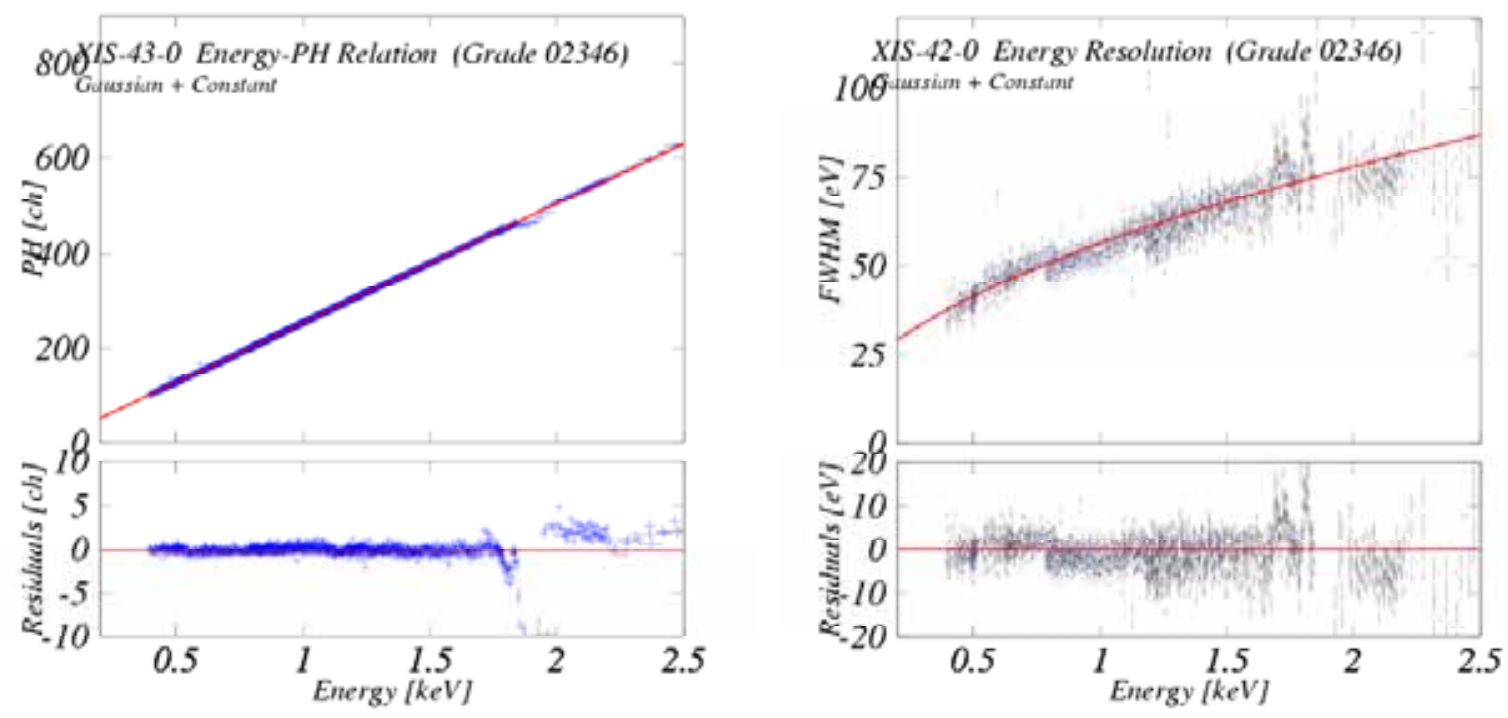

Figure 8. (a) Center of $\mathrm{PH}$ as a function of incident X-ray energy (Ex). The relation below $1.7 \mathrm{keV}$ is well fitted with a straight line with nearly a zero offset. Further examination is needed about the deviation at and above Si-K edge. (b) Energy resolution (FWHM) as a function of incident X-ray energy (Ex). FWHM of about $40 \mathrm{eV}$ at $0.5 \mathrm{keV}$ is typical for the XIS FI-CCDs we measured. We need to refine the data reduction, however, about some gaps in the residual, each of them corresponding to the boundary of XIS CCD quadrants.
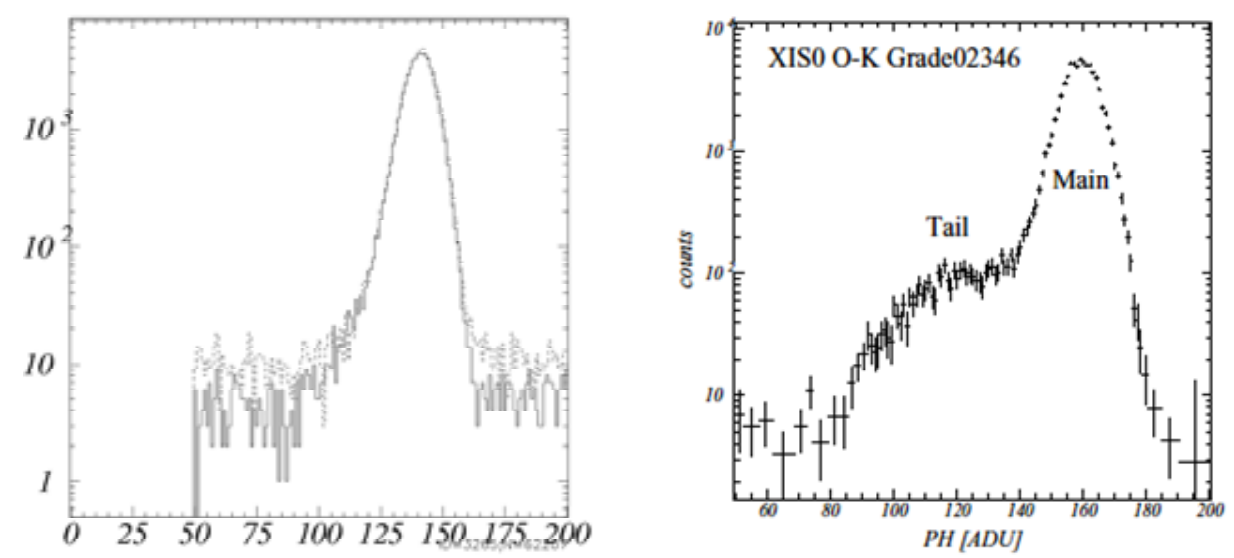

Figure 9. (a) PH distribution of XIS FI-CCD for Astro-E2 against O-K emission line (0.525keV) incidence. Solid line is the data when we set the spplied hight voltage of the soft X-ray source to be $1 \mathrm{kV}$, while the dashed line is that with $5 \mathrm{kV}$. The former case should be free from the contamination from the low energy tail of the higher order light. (b) PH distribution of XIS CCD for Astro-E1 against O-K emission line $(0.525 \mathrm{keV})$ incidence, for comparison. The low energy tail is less evident for Astro-E2 CCD. 


\subsection{Quick look results on BI-CCD}

Calibration procedure for XIS FM BI-CCD cameras is very similar to that for FI-CCD. However, we needed to use different operation parameters in order to accommodate with their higher quantum efficiency at low energy side that that for FI-CCD. We also need to use different data reduction parameters. We tentatively used the event threshold of 20ADU and the split threshold of 7ADU. In the case of FI-CCD fraction of grade 7 events is negligible, while it is not for BICCD. We apparently need to optimize the data reduction parameters, or we may need to introduce other method than the grade method for event extraction. We, however, compare the number of detected events as a function of incident X-ray energy for FI-CCD and for BI-CCD in Fig. ?. It demonstrates huge advantage of BI CCD in low energy quantum efficiency. From this plot, we know the BI CCD quantum efficiency is higher than that of FI CCD by factor of more than 50 at $0.3 \mathrm{keV}$, and about 4 at $0.5 \mathrm{keV}$. The energy resolution of BI CCD is comparable to that of FI CCD, about $45 \mathrm{eV}$ at $0.5 \mathrm{keV}$. With these preliminary results, the XIS BI CCDs are expected to provide unprecedented results in soft X-ray astronomy if they fly.

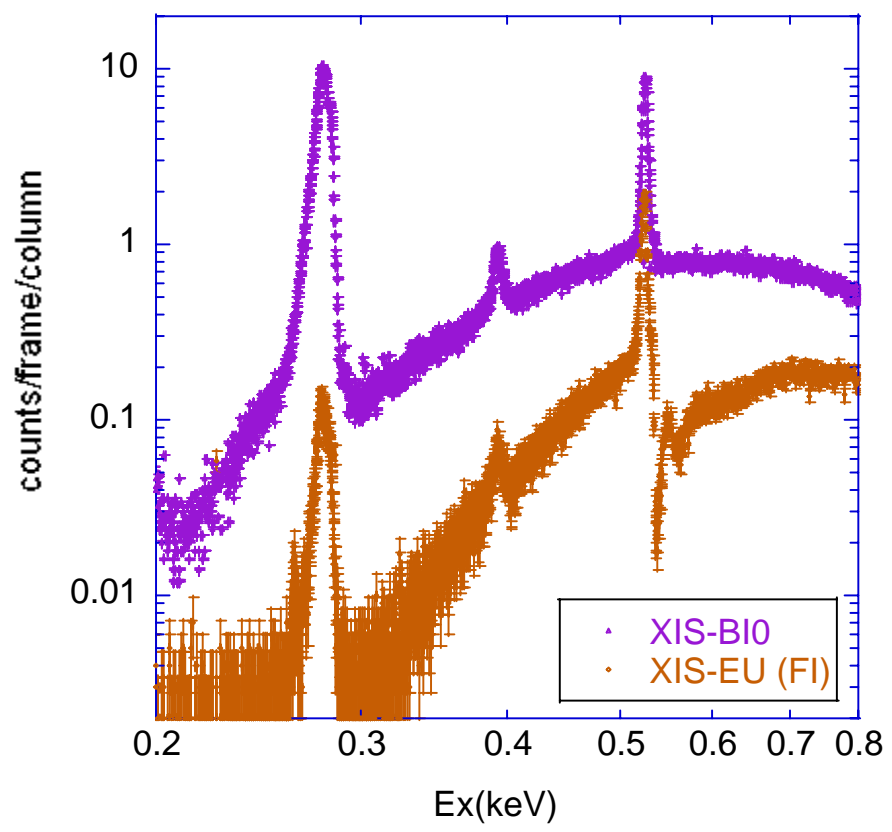

Figure 10. Detected X-ray counting rate for FI-CCD and for BI-CCD. The soft X-ray source was operated with an applied high voltage of $1 \mathrm{kV}$, and the SA grating was used. The ratio of these two almost equals to the relative quantum efficiency of these two CCDs. The BI-CCD has higher quantum efficiency than the FI-CCD by factor of more than 50 at $0.3 \mathrm{keV}$ and about 4 at $0.5 \mathrm{keV}$.

\section{SUMMARY}

We present outline of soft X-ray calibration of Astro-E2 XIS. We have not yet completed the analysis nor experiments, however we could confirm the basic performance of the XIS in soft X-ray energy band are almost what we expected. Four FM XIS cameras, the AE/TCEs for them, and the DE will be installed on the Astro-E2 satellite in July 2004, though we have not yet determined which XIS FM cameras from four FIs and two BIs will be selected. Various kinds of tests, including a thermal vacuum test of the whole Astro-E2 satellite in August 2004, will be performed at ISAS and KSC until the scheduled launch on February 2005. A lot of work is required to refine the analysis of the calibration data and to make response matrices of XIS for general users. The calibration task in the orbit will also be important as for ASCA and the following satellites. 


\section{ACKNOWLEDGMENTS}

Astro-E2 XIS is a Japan-US collaboration project in which many people have participated to design, produce, and test the system. We acknowledge all of the members, especially, M. Bautz, R. Foster, S. Kissel, G. Prigozhin, B. LaMarr (CSR/MIT), B. Burke, A. Pillsbury (LL/MIT), T. Dotani, M. Ozaki, H. Murakami (ISAS/JAXA), E, Miyata (Osaka University), K. Koyama, T. Tsuru, H. Matsumoto, H. Nakajima (Kyoto University), S. Kitamoto (Rikkyo University), H. Awaki (Ehime University) for their great contributions to the project, and also for the supports to our calibration activity at Osaka. We thank all the exmembers in Osaka University XIS team who developed the calibration facilities and software for the Astro-E1 XIS, without which Astro-E2 XIS calibration was impossible. K. Hayashida acknowledges the support from the Grant-in-Aid for Scientific Research (14654047 and 14340061) of the Ministry of Education, Culture, Sports, Science and Technology.

\section{REFERENCES}

1. K. Hayashida, S. Kitamoto, E. Miyata, H. Tsunemi, K. Hashimotodani, K. Katayama, T. Kohmura, R. Asakura, K. Yoshita, H. Katayama, M. Shouho, K. Koyama, T.G. Tsuru, H. Awaki, T. Dotani, M. Ozaki, G.R. Ricker, J.P.Doty, M.W. Bautz, S.E. Kissel, R.F. Foster, "Soft x-ray response of the prototype CCD camera (XIS) for Astro-E," in EUV, X-ray, and Gamma-Ray Instruments for Astronomy IX, O.H.W. Siegmund and M.A. Gummin, eds., Proc. SPIE 3445, pp. 278-290, 1998.

2. K. Hayashida, S. Kitamoto, E. Miyata, H. Tsunemi, K. Yoshita, T. Kohmura, K. Mori, K. Katayama, H. Katayama, M. shouho, M. Ohta, T. Dotani, M. Ozaki, K. Koyama, H. Awaki, T. Tsuru, G. Ricker, M. Bautz, R. Foster, S. Kissel, “X-ray Imaging Spectrometers for Astro-E: Ground Calibration in Soft X-ray Range," in X-ray Optics, Instruments, and Missions III, J.E. Trümper \& B.A. Aschenbach, eds., Proc. SPIE 4012, pp. 123-136, 2000.

3. T. Dotani, M. Ozaki, H. Murakami, K. Koyama, T.G. Tsuru, H. Matsumoto, H. Tsunemi, K. Hayashida, E, Miyata, S. Kitamoto, H. Awaki, M.W. Bautz, J.P. Doty, G.R. Ricker, R.F. Foster, G.Y. Prigozhin, S.E. Kissel, B.E. Burke, A.D. Pillsbury, "X-ray imaging spectrometer (XIS) on board Astro-E2," in X-ray and Gama-Ray Telescopes and Instruments for Astronomy, J.E. Trümper \& H.D. Tananbaum, ed., Proc. SPIE 4851, pp. 1071-1079, 2003.

4. S. Kitamoto, T. Kohmura, N. Yamamoto, H. Saito, H. Takano, K. Suga, E. Ozawa, K. Suzuki, R. Kato, Y. Tachibana, Y. Tsuji, K. Koganei, K. Hayashida, H. Katayama, H. Enoguchi, Y. Nakashima, T. Shiroshoji, "Soft X-ray transmission of optical blocking filters for the X-ray CCD cameras onboard Astro-E 2,” Nucl.Instr. Methods A 505, pp.683-687, 2003.

5. H. Katayama, M. Shouho, T. Kohmura, K. Katayama, K. Yoshita, H. Tsunemi, S. Kitamoto, K. Hayashida, E. Miyata, K. Hashimotodani, K. Koyama, G. Ricker, M.W. Bautz, R. Foster and S. Kissel, "Quantum Efficiency of the CCD Camera (XIS) for the ASTRO-E Mission,” Nucl.Instr. Methods A 436, pp.74-78, 1999.

6. M. Shouho, K. Katayama, H. Katayama, T. Kohmura, H. Tsunemi, S. Kitamoto, K. Hayashida, E. Miyata, K. Hashimotodani, K. Yoshita, K. Koyama, G. Ricker, M.W. Bautz, R. Foster and S. Kissel, "Soft X-Ray Response of the CCD Camera (XIS) for the ASTRO-E Mission,” Nucl.Instr. Methods A 436, pp.85-90, 1999.

7. M. Shouho, "Detection efficiencies of XIS onboard Astro-E," (in Japanese) Master Thesis of Osaka University, 2000. (http://wwwxray.ess.sci.osaka-u.ac.jp/thesis/shouho_master_thesis.pdf)

8. K. Hayashida, T. Shiroshoji K. Fukuda, H. Katayama, "New calibration method for x-ray detection efficiency without using reference counters," in X-ray and Gama-Ray Telescopes and Instruments for Astronomy, J.E. Trümper \& H.D. Tananbaum, eds., Proc. SPIE 4851, pp. 933-944, 2003.

9. K. Mori, M. Shouho, H. Katayama, S. Kitamoto, H. Tsunemi, K. Hayashida, E. Miyata, M. Ohta, T. Kohmura, K. Koyama, M.W. Bautz, R. Foster and S. Kissel, "Measurement of the response characteristics around K absorption edges of dead-layer materials of a charge-coupled device,” Nucl.Instr. Methods A 459, pp.191-199, 2001. 